Discrete Comput Geom 30:181-184 (2003)

DOI: $10.1007 / \mathrm{s} 00454-003-0003-8$

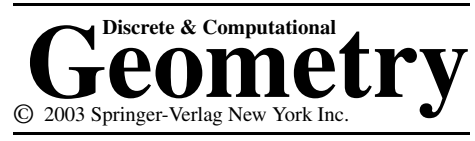

\title{
The Radon Number of the Three-Dimensional Integer Lattice*
}

\author{
Károly Bezdek ${ }^{1}$ and Aart Blokhuis ${ }^{2}$ \\ ${ }^{1}$ Department of Geometry, Eötvös University, \\ Pázmány Péter sétány 1/c, H-1117 Budapest, Hungary \\ kbezdek@ludens.elte.hu \\ ${ }^{2}$ Department of Mathematics and Computing Science, Eindhoven University of Technology, \\ P.O. Box 513, 5600 MB Eindhoven, The Netherlands \\ aartb@win.tue.nl
}

\begin{abstract}
In this note we prove that the Radon number of the three-dimensional integer lattice is at most 17, that is, any set of 17 points with integral coordinates in the threedimensional Euclidean space can be partitioned into two sets such that their convex hulls have an integer point in common.
\end{abstract}

\section{Introduction and Result}

Let $\mathbf{Z}^{d}$ denote the lattice of the points with integral coordinates in the $d$-dimensional Euclidean space $\mathbf{E}^{d}, d \geq 2$. The standard convexity space $\left(\mathbf{Z}^{d}, \mathcal{C}_{d}\right)$ on $\mathbf{Z}^{d}$ is easy to define, namely, the set $C \subset \mathbf{Z}^{d}$ is called convex on $\mathbf{Z}^{d}$, and so it belongs to the family $\mathcal{C}_{d}$ of convex sets on $\mathbf{Z}^{d}$, if there exists a convex set $B$ in $\mathbf{E}^{d}$ such that $C=B \cap \mathbf{Z}^{d}$. From the theory of the convexity space $\left(\mathbf{Z}^{d}, \mathcal{C}_{d}\right)$ we need the following three notions (for more details see, for example, [2]). Let $A$ be an arbitrary subset of $\mathbf{Z}^{d}$ and let $C(A)$ denote the $C$-hull of $A$, that is, let $C(A)=\bigcap\left\{C \in \mathcal{C}_{d} \mid A \subset C\right\}$. Then the Caratheodory number $c(d)$ of the convexity space $\left(\mathbf{Z}^{d}, \mathcal{C}_{d}\right)$ is the smallest positive integer $n$ with the property that for any set $A \subset \mathbf{Z}^{d}$ one has that $C(A)=\bigcup\left\{C\left(A^{\prime}\right) \mid A^{\prime} \subset A\right.$ and $\left.\operatorname{card}\left(A^{\prime}\right) \leq n\right\}$. The Helly number $h(d)$ of the convexity space $\left(\mathbf{Z}^{d}, \mathcal{C}_{d}\right)$ is the smallest positive integer $n$ with the property that if $\mathcal{H} \subset \mathcal{C}_{d}$ is an arbitrary finite family of convex sets on $\mathbf{Z}^{d}$ such that any $n$ sets of $\mathcal{H}$ intersect, then $\bigcap \mathcal{H}$ is nonempty. If $X$ is an arbitrary subset of $\mathbf{Z}^{d}$ with the partition $(Y, Z)$ (i.e., $Y$ and $Z$ are disjoint with $\left.Y \cup Z=X\right)$ such that

* Károly Bezdek was partially supported by the Hungarian National Science Foundation (OTKA), Grant No. T029786, and by the Combinatorial Geometry Project of the Research Foundation FKFP0151/1999. 
$C(Y) \cap C(Z)$ is nonempty, then the partition $(Y, Z)$ is called a Radon partition of $X$ on $\mathbf{Z}^{d}$. Finally, the Radon number $r(d)$ of the convexity space $\left(\mathbf{Z}^{d}, \mathcal{C}_{d}\right)$ is the smallest positive integer $n$ with the property that any set $X \subset \mathbf{Z}^{d}$ of cardinality $\operatorname{card}(X) \geq n$ possesses a Radon partition on $\mathbf{Z}^{d}$.

It is easy to see that $c(d)=d+1$. It is much harder to determine $h(d)$. Doignon [1] was the first who proved that $h(d)=2^{d}$. The problem of determining the Radon number $r(d)$ of the integer lattice $\mathbf{Z}^{d}$ was raised by Onn [2], who proved that $r(2)=6$ and $1+5 \cdot 2^{d-2} \leq r(d) \leq d\left(2^{d}-1\right)+3$, for all $d \geq 3$, in particular, $11 \leq r(3) \leq 24$. In this note we improve the later result of Onn [2] as follows.

Theorem. The Radon number of the three-dimensional integer lattice $\mathbf{Z}^{3}$ is at most 17 , that is, $r(3) \leq 17$.

We close the introduction with an easy proof of $r(2) \leq 6$, which is different from the proof in [2] and also motivates our proof of the Theorem. The proof is indirect, so assume that there are six points of $\mathbf{Z}^{2}$ that have no Radon partition on $\mathbf{Z}^{2}$. Then they must lie in convex position and so we can label them according to some orientation of $\mathbf{E}^{2}$ as $\mathbf{p}_{1}, \mathbf{p}_{2}, \ldots, \mathbf{p}_{6}$. Let $P=\left\{\mathbf{p}_{1}, \mathbf{p}_{2}, \ldots, \mathbf{p}_{6}\right\}$ and $P_{i}=P \backslash\left\{\mathbf{p}_{i}\right\}$ for all $1 \leq i \leq 6$. As $h(2)=4$ and any four of the sets $P_{i}, 1 \leq i \leq 6$, have a lattice point of $\overline{\mathbf{Z}^{2}}$ in common, we get that there exists a lattice point $\mathbf{p} \in \mathbf{Z}^{2}$ such that $\mathbf{p} \in\left(\bigcap_{1 \leq i \leq 6} C\left(P_{i}\right)\right)$. However, then the triplets $\left\{\mathbf{p}_{1}, \mathbf{p}_{3}, \mathbf{p}_{5}\right\}$ and $\left\{\mathbf{p}_{2}, \mathbf{p}_{4}, \mathbf{p}_{6}\right\}$ form a Radon partition of $P$ with $\mathbf{p} \in C\left(\left\{\mathbf{p}_{1}, \mathbf{p}_{3}, \mathbf{p}_{5}\right\}\right) \cap C\left(\left\{\mathbf{p}_{2}, \mathbf{p}_{4}, \mathbf{p}_{6}\right\}\right)=\bigcap_{1 \leq i \leq 6} C\left(P_{i}\right)$, a contradiction.

\section{Proof of the Theorem}

Our proof is indirect, so we assume that there exists a pointset of 17 lattice points of $\mathbf{Z}^{3}$, say, $X=\left\{\mathbf{x}_{1}, \mathbf{x}_{2}, \ldots, \mathbf{x}_{17}\right\} \subset \mathbf{Z}^{3}$ with no Radon partition on $\mathbf{Z}^{3}$. From this it follows that the points of $X$ are in convex position, that is, every $\mathbf{x}_{i}$ is a vertex of the convex hull of $X$, where $1 \leq i \leq 17$. Then let $X_{i, j}=C\left(X \backslash\left\{\mathbf{x}_{i}, \mathbf{x}_{j}\right\}\right)$ for all $1 \leq i<j \leq 17$. As the Helly number of $\mathbf{Z}^{3}$ is 8 and any eight of the sets $X_{i, j}$ intersect, we find a lattice point $\mathbf{p}$ of $\mathbf{Z}^{3}$ that belongs to all $X_{i, j}$, that is, $\mathbf{p} \in\left(\bigcap_{1 \leq i<j \leq 17} X_{i, j}\right)$.

Now, let $Q=\{Y \subset X \mid \operatorname{card}(Y)=4$ and $\mathbf{p} \in C(Y)\}$. The quadruples $Y \in Q$ will be called blocks. The most basic properties of blocks are summarized in the following claim.

\section{Lemma 1.}

(1) Every two blocks intersect.

(2) For every pair $\left\{\mathbf{x}_{i}, \mathbf{x}_{j}\right\} \subset X$ there exists a block disjoint from it.

(3) The exchange property: for every block $Y$ and every point $\mathbf{x}_{i} \in(X \backslash Y)$ there is a unique block containing $\mathbf{x}_{i}$ and intersecting $Y$ in precisely three points.

Proof. (1) Every two blocks intersect since two disjoint blocks would give rise to a Radon partition of $X$ on $\mathbf{Z}^{3}$. 
(2) For every pair $\left\{\mathbf{x}_{i}, \mathbf{x}_{j}\right\} \subset X$ we have that $\mathbf{p} \in X_{i, j}$ and so there must be a quadruple $Y \subset\left(X \backslash\left\{\mathbf{x}_{i}, \mathbf{x}_{j}\right\}\right)$ with $\mathbf{p} \in C(Y)$. Then $Y$ is the block disjoint from $\left\{\mathbf{x}_{i}, \mathbf{x}_{j}\right\}$.

(3) The four tetrahedra obtained by forming the convex hull of $\mathbf{x}_{i}$ and any three points of $Y$ cover the tetrahedron spanned by the points of the block $Y$. Thus, the point $\mathbf{p}$ lies in exactly one of these tetrahedra, unless it is on a common boundary of two (or more) of them, and in that case it is in the convex hull of a triple $\left\{\mathbf{x}_{i}, \mathbf{x}_{j}, \mathbf{x}_{k}\right\} \subset Y$. However, then it is easy to check that (2) implies that $\left\{\mathbf{x}_{i}, \mathbf{x}_{j}, \mathbf{x}_{k}\right\}$ and $X \backslash\left(\left\{\mathbf{x}_{i}, \mathbf{x}_{j}, \mathbf{x}_{k}\right\}\right)$ form a Radon partition of $X$, a contradiction.

Let $Y$ be an arbitrary block. Then to every point $\mathbf{x}_{i} \in(X \backslash Y)$ there corresponds a point $\mathbf{x}_{j} \in Y$ by the exchange property. Since there are 13 points in $X \backslash Y$ there must be a point $\mathbf{x}_{k} \in Y$ corresponding to at least four points in $X \backslash Y$. Now, consider $T=Y \backslash\left\{\mathbf{x}_{k}\right\}$. We call such a triple $T$ a trio. The most basic properties of trios are summarized in the following claim.

\section{Lemma 2.}

(1)* Every trio is in at least five blocks and every two trios intersect.

(2)* For every pair $\left\{\mathbf{x}_{i}, \mathbf{x}_{j}\right\} \subset X$ there is a trio disjoint from it.

(3)* The trios cover at most seven points of $X$.

(4)* If $\mathbf{x}_{m} \in X$ is a point not covered by any trio and $T$ is a trio, then $\left\{\mathbf{x}_{m}\right\} \cup T$ is a block.

Proof. (1)* Let $Y$ be a block with a point $\mathbf{x}_{k} \in Y$ corresponding to at least four points of $X \backslash Y$, say, $\mathbf{x}_{1}, \mathbf{x}_{2}, \mathbf{x}_{3}, \mathbf{x}_{4}$, via the exchange property. Now, consider the trio $T=Y \backslash\left\{\mathbf{x}_{k}\right\}$. Then $T \cup\left\{\mathbf{x}_{l}\right\}$ is a block for all $l=1,2,3,4$. So, $T$ is in at least five blocks. Using (1) and the first part of (1)* one immediately obtains that every two trios must intersect.

$(2)^{*}$ This follows from (2) in a straightforward way.

(3)* Let $T=\left\{\mathbf{x}_{i}, \mathbf{x}_{j}, \mathbf{x}_{k}\right\}$ be a trio and based on (1)* and (2)* let $T_{i}, T_{j}, T_{k}$ be trios with $T \cap T_{i}=\left\{\mathbf{x}_{i}\right\}, T \cap T_{j}=\left\{\mathbf{x}_{j}\right\}, T \cap T_{k}=\left\{\mathbf{x}_{k}\right\}$. Since $T_{i}, T_{j}, T_{k}$ have to intersect each other $5 \leq \operatorname{card}\left(T_{i} \cup T_{j} \cup T_{k}\right) \leq 7$. In what follows we deal only with the case $\operatorname{card}\left(T_{i} \cup T_{j} \cup T_{k}\right)=7$ and leave the remaining cases, that can be treated similarly, to the reader. The assumption $\operatorname{card}\left(T_{i} \cup T_{j} \cup T_{k}\right)=7$ implies that there is a point $\mathbf{x}_{l}$ with $\mathbf{x}_{l}=T_{i} \cap T_{j} \cap T_{k}$. Now, assume that there is a point $\mathbf{x}_{m}$ different from the points of $T_{i} \cup T_{j} \cup T_{k}$ covered by some trio $T_{m}$. As every two trios must intersect card $\left(T \cap T_{m}\right)=1$, say, $T \cap T_{m}=\left\{\mathbf{x}_{i}\right\}$. However, then $T_{m}=\left\{\mathbf{x}_{i}, \mathbf{x}_{l}, \mathbf{x}_{m}\right\}$. Finally, according to (2)* there is a trio $T_{n}$ that is disjoint from the pair $\left\{\mathbf{x}_{i}, \mathbf{x}_{l}\right\}$. As $T_{n}$ has to intersect $T_{m}$ as well as $T_{i}=\left\{\mathbf{x}_{i}, \mathbf{x}_{l}, \mathbf{x}_{n}\right\}$ it follows that $\left\{\mathbf{x}_{m}, \mathbf{x}_{n}\right\} \subset T_{n}$. However, then there is no choice for the third element of the trio $T_{n}$ such that $T_{n}$ intersects $T, T_{j}$ as well as $T_{k}$, a contradiction.

(4)* As $T$ is in at least five blocks and the trios cover at most seven points there is a block $Y=T \cup\left\{\mathbf{x}_{n}\right\}$ with $\mathbf{x}_{n} \in X$ not covered by any trio. If $\mathbf{x}_{n}=\mathbf{x}_{m}$, then we are done. If $\mathbf{x}_{n} \neq \mathbf{x}_{m}$, then applying the exchange property to the point $\mathbf{x}_{m}$ and the block $Y$ we get that $T \cup \mathbf{x}_{m}$ is a block.

Let $X^{\prime} \subset X$ be the union of the trios and let $\mathbf{x}_{i} \in X$ be a point not covered by any trio, that is, let $\mathbf{x}_{i} \in\left(X \backslash X^{\prime}\right)$. Then let $H$ be a plane in $\mathbf{E}^{3}$ that strictly separates $\mathbf{x}_{i}$ from 
$\left(X \backslash\left\{\mathbf{x}_{i}\right\}\right) \cup\{\mathbf{p}\}$. Project $X^{\prime}$ and $\mathbf{p}$ from $\mathbf{x}_{i}$ onto $H$. Denote this central projection by *. Note that every trio forms a block together with $\mathbf{x}_{i}$ having $\mathbf{p}$ in the three-dimensional interior of the convex hull of the given block in $\mathbf{E}^{3}$ (see properties (3) and (4)*), so in $H$ we find $\mathbf{p}^{*}$ in the two-dimensional interior of the convex hull of the image of each trio. Notice that every line through $\mathbf{p}^{*}$ contains at most one point of $X^{\prime *}$.

Now, in the plane $H$ draw an arbitrary circle with center $\mathbf{p}^{*}$ and radially project the points of $X^{* *}$ on this circle. Then we still have the property that trios produce triangles containing the center $\mathbf{p}^{*}$ in their two-dimensional interiors. Thus, (2)* implies that there is no diameter of the circle with just two points of $X^{\prime *}$ on one side. This immediately shows that card $X^{\prime} \geq 7$, so with (3)* this yields that card $X^{\prime}=7$.

We are now in a position to finish the proof of the Theorem.

We have seven points on a circle, no two antipodal, and every diameter not containing one of them separates them in groups of size 3 and 4 . If we label these points 0,1 up to 6 according to some orientation of the circle we see that there are two kinds of triangles containing the center, namely, 014 and 024 or their cyclic shifts $(\bmod 7)$.

Now, suppose that 014 is a trio. Then there must be a trio disjoint from 01 and this can only be 246 since it has to contain 4 . Hence 125 cannot be a triple, since this would force 350 , disjoint from 246 . So the only trio disjoint from 06 can be 145 producing also 136. Finally, the trio disjoint from 34 has to be 256 , producing 024 , but then we have two disjoint trios 024 and 136, a contradiction. So we see that triples like 014 do not occur, and all triples are cyclic shifts of 024 . This quickly leads to a contradiction and completes the proof of the Theorem.

\section{References}

1. J. P. Doignon, Convexity in crystallographical lattices, J. Geom. 3 (1973), 71-85.

2. S. Onn, On the geometry and computational complexity of Radon partitions in the integer lattice, SIAM J. Discrete Math. 4(3) (1991), 436-447. 